\title{
LA VIVIENDA RURAL. \\ SU COMPLEJIDAD Y ESTUDIO DESDE DIVERSAS DISCIPLINAS.
}

\author{
CONCEPCIÓN SÁNCHEZ QUINTANAR ${ }^{1}$ y ERIC ORLANDO JIMÉNEZ ROSAS ${ }^{2}$
}

Manizales, 2009-12-14 (Rev. 2010-05-30).

\section{RESUMEN}

La comprensión de la lógica de la vivienda rural (VR) aumenta con la aportación de estudios multidisciplinarios, dada la complejidad de sucesos que en ella se desarrollan. Por un lado, se deben conocer las actividades agrícolas a que se dedica la familia y los ecosistemas en donde se ejecutan; y por otro, las relaciones intra familiares y las externas que establecen con familias similares, con las que se forman las redes sociales de las comunidades, y la cultura que rige sus comportamientos familiares y sociales.

El análisis bibliográfico y documental que se presenta en este escrito, identifica contribuciones teóricas y empíricas, que reflejan el interés y avances en el estudio de la VR por parte de diferentes disciplinas: Estudios de ciencia básica aportan conceptos, definiciones y clasificaciones; las contribuciones de aplicación, se orientan hacia el bienestar de la familia en su vivienda y algunas están conceptualmente asociadas con la calidad de vida y la sustentabilidad.

De la clasificaciòn y anàlisis la principal aportaciòn es un modelo de Calidad de Vida para la la VR, cuya base estructural es el modelo de Desarrollo Humano de Binfenbreiner, (2002). Esta contribuciòn señala relaciones horizontales entre las similitudes de temàticas estudiadas por diferentes disciplinas e investigaciones revisadas. De manera vertical indica las relaciones entre niveles ecològicos diferentes, el microsistema es la familia y la VR, el mesosistema las relaciones entre las familias o comunidades, y el exosistema la demarcaciòn territorial. La sistematizacipon e indicadores del modelo pueden servir para el ordenamiento o reordenamiento territorial.

PALABRAS CLAVE:

Vivienda rural, análisis bibliográfico, calidad de vida.

\section{RURAL HOUSING. ITS COMPLEXITY AND STUDY FROM THE PERSPECTIVE OF SEVERAL DISCIPLINES.}

\begin{abstract}
In order to obtain an understanding of the rural housing (RH) logic, a multi and interdisciplinary approach is essential, given the complexity of its events. First off, it's necessary to incorporate the agricultural activities and the ecosystems associated to such actions. Also, it's important to consider the intra-family relationships and the relations with other similar families, with which social networks of communities, and the culture that configure their family and social behavior. The bibliographic and documental analysis permitted the identification of theoretical and empirical contributions that reflect the research progress on $\mathrm{RH}$ carried out by diverse disciplines: Basic science studies provide concepts, definitions and classifications, as well as applied contributions that are mainly
\end{abstract}


oriented towards the family's well-being in the housing, and some are conceptually associated with quality of life and sustainability. The main conclusion was a multidisciplinary model of quality of life for $\mathrm{RH}$, with a basis on the Human Development proposed by Bonfenbrenner (2002). The model shows the horizontal relationships between similar topics studied by different discipline and researches. In a vertical manner, the model indicates ecological relations, the family and $\mathrm{RH}$ as the microsystem, the relations between families and communities are the mesosystem; while the exosystem is composed of territorial demarcation Additionally, the model's systematization and indicators could be implemented for territorial planning.

KEY WORDS: rural housing, bibliographic analysis, quality of life.

\section{INTRODUCCIÓN}

Para el estudio de los espacios y las relaciones que las personas establecen con ellos, la Psicología Ambiental hace una diferenciación entre espacios no construidos y construidos; estos últimos pueden ser externos e internos. La vivienda rural (VR) es un espacio construido, con una parte interna y otra externa. Por las actividades agropecuarias que realiza la familia, la vivienda se encuentra inmersa en ecosistemas naturales que cultiva, conserva, transforma o deteriora.

En este trabajo se considera a la VR como: un organismo eminentemente activo e interactivo con el medio natural, construido y comunitario, que constituye una herencia, no sólo cultural, sino también de sostén emocional y cohesivo de las familias, apoyado o con gran influencia de sus actividades económicas y comunitarias; este comportamiento contrasta con el que se produce en las viviendas de las ciudades, cuyas actividades ya no fomentan tales características en la familia (Sánchez,

La percepción de la lógica de la VR, requiere, sin lugar a dudas, un enfoque y marco multi e interdisciplinario, ya que se requiere comprender las labores agrícolas en determinados ecosistemas, las relaciones internas y externas de las familias que viven en ellas, y sus relaciones con otras familias, que en conjunto construyen las redes del tejido social de las comunidades rurales. Estas tienen influencias muy diversas en lo social, económico y cultural; sus situaciones varían también de acuerdo a la zona ecológica y demográfica, y a la economía regional y nacional. Además hay dos situaciones transversales que viven las comunidades rurales: la emigración al exterior y nacional, y el aumento de la pobreza, sobre todo en zonas marginales de paupérrimas condiciones ecológicas.

\section{Calidad de vida en la vivienda rural}

El concepto de calidad de vida (CV) se generó como medida para buscar niveles aceptables de condiciones para la vida humana; sus múltiples aportaciones tienen el sesgo de aplicabilidad inmediata para promover la restauración de condiciones de vida sana y permisible en la población, por lo que la revisión bibliográfica puede ser muy amplia si no se establecen límites. En esta investigación se citan los trabajos más significativos que son fuentes de conocimiento y reflexión, para su aplicación en diferentes ámbitos de la vida humana. 
El carácter multidisciplinario en el estudio de la CV presenta una limitante que ha sido la elaboración de instrumentos confiables que midan y comparen el concepto en diferentes países, lo que significa diferentes sociedades, culturas, ecologías y niveles socioeconómicos; y, desde luego, diferentes enfoques en la investigación (Veenhoven, 2005). Superar el problema promovió la comparación entre naciones, expresando la familiaridad o lejanía que pueden tener unas con relación a otras, así como las características únicas de cada país. En una investigación dirigida por el mismo autor en 67 países, emergió la subjetividad de los sujetos como inevitable, porque sólo ellos pueden apreciar cómo viven. Sin duda fue un aporte importante, dado que los indicadores empleados eran, para países desarrollados, los ingresos y los servicios con los que contaban las personas; para países pobres, se empleaban los indicadores de salud, nutrición, mortandad y acceso a fuentes de trabajo.

Al estudiar la CV se han elegido diversos sinónimos: "salud y bienestar", "satisfactores económicos o de otro tipo" y "ser felices". En Estados Unidos, Kenneth, Land, Lamb y Taylor (2007), usaron el concepto de "bienestar de niños y jóvenes" para estudiar los indicadores de CV concernientes a esas etapas de desarrollo. Con la información que obtuvieron, elaboraron en 1975 el cuestionario Well-Being Index (WBI) para esas edades. Sus investigaciones entre 1975-2004 en niños y jóvenes les permitieron conocer los cambios en el significado de "bienestar" por generaciones e interrelacionarlos con las épocas socioeconómicas y políticas de ese país.

En el área de la salud se estudia la percepción o las respuestas subjetivas sobre el ambiente local, porque la persona, al interiorizar los factores sociales externos como conocimiento, relaciona el ambiente externo (social, cultural y físico) con el interno (biológico). Muhajarine, Labonte, Williams y Randall (2008) han considerado la percepción del individuo sobre sí mismo, con su vivienda y comunidad.

En México, Landázuri, Terán, Mercado y Sánchez (2003) refieren el concepto de $\mathrm{CV}$ a la vivienda como factor que incide directamente en la cualidad de modo de vida de sus habitantes; esto es entendible desde la sensación y la percepción que cada individuo tiene del grado de satisfacción que le aporta su vivienda para la resolución de sus necesidades.

\section{OBJETIVOS}

Por lo anterior, los objetivos de este trabajo son:

1. Proporcionar información multidisciplinaria de los estudios que el tema ha generado y un Modelo que permita visualizar las relaciones entre disciplinas y los temas de interés sobre la VR. Esto sin duda facilitará la orientación de profesionales interesados en su comprensión desde diferentes dimensiones.

2. Obtener indicadores desde la perspectiva multidisciplinaria, para describir la calidad de vida que la VR produce a sus moradores.

El concepto de multidisciplina que guió el trabajo surge de la necesidad de delimitar campos de conocimiento, acción y método. La multidisciplina se presentó como "la acción conjunta de varias disciplinas para resolver un problema en el ámbito práctico, en donde es tan importante la explicación del 
problema, como las acciones coordinadas para resolverlo" (Cardoso, 1999, p. 34). En este trabajo hacemos extensivo estas consideraciones a determinadas acciones sistemáticas y metodológicas cuya base epistemológica proviene de diferentes disciplinas, como pudiera ser el caso de Trabajo Social, Enfermería, Administración o Demografía, entre otras. Esta decisión se hizo necesaria para incluir aportaciones importantes de profesionales dedicados más a la acción y el trabajo social que a la investigación; no obstante que en situaciones determinadas se les hace necesario incluirse en la investigación de carácter aplicado para resolver problemas específicos.

\section{MÉTODO}

\section{Población rural}

En cuanto a la delimitación entre la población urbana y la rural, no existe un punto donde desaparezca lo urbano y comience lo rural, y por ello la división resulta arbitraria (Villalvazo, Corona \& García, 2002). La ONU considera que cuando se realizan comparaciones entre países de diferente densidad poblacional y grado de desarrollo, el número de habitantes no aporta claridad. Los criterios de Unikel, Ruiz y Garza (1976), son más flexibles, para ellos una localidad es rural cuando tiene máximo 5000 habitantes, y mixta cuando son entre 5000 y 15000, y según la proporción de actividad económica primaria de la familia que vive en el campo.

En la realidad los asentamientos humanos, sean clasificados o no por el número de habitantes, tienen relaciones entre ellos y movimientos que les permite soslayar las delimitaciones geográficas y sociales. El concepto de nueva ruralidad que se debatió a finales de la década de los años de 1990 y principios de la del 2000, argumentan que las zonas rurales marcan un territorio en el que la población tiene más actividades económicas y sus culturas permean a las ciudades, y se establecen relaciones de interdependencia (E. Pérez, 2001). Esas relaciones tienen sus particularidades, históricas, sociales, culturales y ecológicas, que definen su realidad propia. Llambí (1995) plantea que estos procesos aparecen en el mundo de manera simultánea e interrelacionados con los procesos globalizadores y que tienden a reestructuraciones geoeconómicas y geopolíticas, que se escapan al estudio disciplinario.

Avanzados los procesos globalizantes, con las complicaciones de la sobrepoblación y los problemas climáticos, en muchas zonas ya no hay una diferencia visible o fácilmente perceptible entre una población y otra. Ahora es la población urbana que no sólo se relaciona con la rural, sino que la invade y se establece en regiones que antes eran productivas. La expansión de las grandes ciudades se realiza sobre los campos agrícolas, convirtiéndolos en espacios de construcción urbanos en especial de interés social y grandes centros comerciales.

No obstante, son distinguibles las familias rurales quienes conservan actividades agropecuarias en alguna medida, y complementan sus ingresos con trabajo asalariado o venta de productos agrícolas con algún valor agregado. Estas distinciones son uno de los objetivos censales de los países que requieren comprender los movimientos demográficos. Del mismo modo, zonas rurales son distinguibles por un conjunto de características que las distancian de las zonas urbanas: menor extensión y población, así como menor cantidad de servicios y equipamientos. 


\section{Análisis bibliográfico y documental}

La búsqueda bibliográfica abarcó las décadas de 1998 a 2008, en las siguientes bases de datos: a) Isi Web of Science, ProQuest, Ebsco Host, Jstor, Wiley Blackwell, Science Direct, Springer Link, E-brary, Sage Journals, Scirus, PsycINFO, Elsevier; Ansi Journals; b) reportes de investigación, memorias de congresos, revistas locales y tesis; y c) investigaciones que se han realizado en el Colegio de Posgraduados en Ciencias Agrícolas.

El análisis cualitativo del material documental se organizó con el software EndNote X (Thomson ISI ResearchSoft, 2004); se clasificó tomando en consideración las disciplinas predominantes de los documentos y las temáticas de interés.

\section{RESULTADOS}

Los resultados se dividen en dos apartados, el primero, de análisis cualitativo, presenta cuatro tipos de categorías en que se concentran los aportes de las investigaciones y estudios analizados, y el segundo es un Modelo de Calidad de Vida para la VR:

\section{Análisis cualitativo}

\subsection{Aportes de investigaciones básicas}

\section{Investigaciones históricas}

La historia de la vivienda identifica una etapa de evolución de la humanidad, caracterizada por la separación de las labores agropecuarias de los espacios habitacionales, los cuales son derivados de costumbres y tradiciones que dan cuerpo a lo doméstico, a lo íntimo y a lo privado, dedicando otros espacios para lo público; este momento se ubica en el Medioevo, cuando la alta burguesía deseaba confort y eliminó las actividades económicas de la casa y del predio familiar (Rybczynski, 1986).

Una etapa histórica puede relacionar contextos, causas y modificaciones de las viviendas. Los contrastes sociales, étnicos, económicos y políticos de México previos a la revolución de 1910 se reflejaron en las viviendas. Los latifundios influenciados por el extranjero separaban la habitación de los espacios productivos; y las chozas prehispánicas indígenas mostraban menos cambios y mayor adaptabilidad ecológica (Bolis, 1982).

También en México, la arquitectura registra un cambio de actitud histórico, del menosprecio al aprecio de la VR y la vernácula. El inicio data de finales del siglo XVIII y principios del XIX (Viollet-le-Duc, 1874 citado por Aguilar, 2001); entre 1960 y 1970 la arquitectura se orientó hacia la VR (Aguilar, 2001), que al inicio se asociaba a la pobreza (Fathy, 1969 citado por Aguilar, 2001), pero los resultados resaltaron la sabiduría y coherencia con la que los usuarios resuelven los problemas de sus viviendas, así como la dificultad de lograr diseños coherentes sin considerar los valores constantes de los mismos (Rapoport, 1969 citado por Aguilar, 2001). Entre 1970 y 1980 se difunde el interés por la VR y la vernácula, en cuanto a materiales de construcción de las propias regiones; para su estudio, 
se publica la Enciclopedia mundial de arquitectura vernácula con trabajos de 700 autores de 80 países (Oliver, 1997 citado por Aguilar, 2001).

Un problema actual son los cambios en el ambiente rural que provocan la sobrepoblación de las ciudades medianas y grandes, cuya periferia se une a la superficie rural y forma las zonas metropolitanas (Rybczynski, 1986). La urbanización acelerada ha hecho que las ciudades ocupen el $4 \%$ de la superficie del planeta y alojen a la mitad de su población, quienes consumen tres cuartos de los recursos naturales y generan otra cifra igual de contaminación y desechos (Redman \& Jones, 2005). En 1957, el 30\% de la población global vivía en ciudades, mientras que en el año 2000 el $47 \%$, y para 2030 se espera que sea el $60 \%$. En las ciudades de países desarrollados vive el $75 \%$ de la población mundial, y para 2030 se calcula que sea el 83\%; también se espera que el mayor crecimiento de población urbana se dé en países en desarrollo (UN Population Division, 2003 citado por Redman \& Jones, 2005). La sobrepoblación es la principal causa del daño ecológico, el crecimiento de la inseguridad y la necesidad de lograr el desarrollo sustentable (Cassils, 2004). también es particularmente productora de contaminación, dando como resultado que los ecosistemas lleguen a sus límites al consumir sin medida agua y suelo (Harte, 2007).

\section{Conceptualizaciones de vivienda rural}

Los conceptos que se transcriben aportan conocimientos sobre la VR y revelan abstracciones de su esencia de acuerdo a diferentes disciplinas.

Hay una fuerte influencia de las ciencias sociales en arquitectos y agrónomos, la conceptualización sobre la vivienda hecha por Heidegger (1992) en la que se basan Correa (2000) y Mellace (2000) es un lugar central de la existencia humana, donde la relación trabajo-producción-vida familiar está en clara interacción con el entorno, no sólo comprende la unidad de habitación, también el espacio de producción, la diseñan, construyen y modifican sus moradores con técnicas tradicionales, auto-producción de materiales y componentes básicos.

Sámano et al. (2001) enfatizan que es necesario concebir a lo rural y lo urbano como un complejo sistema de relaciones de intercambio, transferencias e interpenetraciones, en un ámbito no sólo nacional sino internacional. Paredes (2000) encuentra que en los espacios correspondientes a actividades domésticas y productivas de la VR se reflejan los usos, disponibilidades sociales y culturales, como la educación y la religión. La vivienda se conceptualiza de diferentes maneras porque a medida que evolucionan los seres humanos, ella también lo hace (Rybczynski, 1986).

Chayanov (1974) en la economía y Parsons (1975) en la sociología coinciden en que la familia es una unidad natural, institución universal basada en su reproducción biológica y social. El primero explica la división del trabajo considerándola Unidad Económica Campesina, basada en la asignación natural del trabajo: mujeres en actividades reproductivas biológicas y sociales, hombres en las productivas y de intercambio; e identifica los espacios internos y externos de la VR correspondientes. Parsons ve la parte social derivada de la coresidencia y las formas estructurales y funcionales que toman las familias en la cultura campesina.

De los conceptos "familia", "hogar" y "casa" que Parsons aporta a la sociología, Parada (1993) ve diferentes elementos que interactúan en la VR: la coresidencia, el matrimonio (contrato conyugal), las relaciones de poder entre 
géneros, el trabajo doméstico, la sexualidad, la procreación, el ciclo de vida, las definiciones de actividades o roles por género, las relaciones de parentesco, y las relaciones económicas. En términos de funcionalidad, la VR se relaciona con la actividad productiva y la cultura de sus habitantes porque es uno de los medios de trabajo (Damián, 1991). La asociación de espacios, funciones y roles, ha permitido la comprensión de la lógica de la VR para la familia que la habita (Monterrubio, 2000). En forma conceptual, la VR es un espacio construido por la actividad consciente del hombre que ocurre en la humanización de la interacción hombre-naturaleza; es el espacio indispensable para las relaciones primarias o nucleares (Vargas, 2000).

\section{Descripciones de viviendas}

La VR incluye habitación y áreas productivas, ya que hay actividades que se realizan dentro con participación de varios o todos los miembros de la familia; su ubicación en los asentamientos rurales se relaciona con la accesibilidad y distancia adecuada a las tierras de cultivo. También es un espacio cultural, ritual de saber, porque ocupa un lugar central para las actividades ceremoniales, de sociabilidad y de relaciones y solidaridad comunitarias (Roze, 2000). La ocupación de la tierra y la distribución de funciones del trabajo campesino constituye la estrategia básica para la organización espacial de la vivienda en ambientes interiores y exteriores, que constituyen el escenario principal de la vida familiar y concentran el $90 \%$ de las actividades de grupo (Rotorando \& Mellase, 2000), descripción que se hace extensiva a Iberoamérica.

Al describir la VR por sus valores, Villar (2001) señala que es útil porque cada espacio es adecuado para las necesidades del habitante y su construcción requiere elementos mínimos. Es congruente con la imagen de su creador, existe concordancia entre sus elementos: material-apariencia, forma-función, formautilidad, forma-historia, y con el medio natural. Es estética por lo conveniente para sus moradores, además contiene elementos naturales construidos, armónicos y agradables a la vista; es social porque es la representación de una cultura, de un conjunto de individuos. Por todo lo anterior, el autor considera a la VR como arquitectura con valor.

La imagen de la VR más difundida es la de campesinos, y la de indígenas, que por lo general están aisladas y dispersas en la sierra, desconocidas y sólo identificables estadísticamente tras desastres naturales (González, 2001). Esas características son un patrón de sociedades indígenas, originadas como estrategia de sobrevivencia y protección ante la colonización española. En las descripciones folclóricas aparecen ideas asociadas a la presencia de animales, agricultura, elementos rústicos y precariedad (Grama, 2000). La VR es autogestionada, autopromovida, autoadministrada y autoconstruida (Vargas, 2000; González, 2001).

\section{Clasificaciones de viviendas rurales}

Las clasificaciones reportadas derivan de conceptos o definiciones de la VR; algunas son categorizaciones de atributos y otras, identificación de elementos.

En cuanto a la tecnología, la VR tradicional y la transicional emplean técnicas artesanales y materiales naturales del entorno (Rotorando \& Mellace, 2000). González (2001) coincide pero agrega la VR moderna que incorpora materiales industriales, mezclando componentes y técnicas tradicionales; aun manteniendo el adobe, su construcción lleva más materiales industrializados. En México, Torres (2001) da cuatro criterios arquitectónicos: conjunto (volúmenes y 
organización de los espacios), distribución (ordenación de los espacios y funciones), elementos (techumbres, muros, puertas y ventanas), y sistemas constructivos (estructuras). Por criterios económicos y antropológicos, Roze (2000) clasifica la VR en: la natural, que facilita las relaciones sociales, de convivencia familiar y las funciones de sus miembros; la de mercancía, por su valor en el mercado; la de producción, por el sistema de trabajo del que participa la familia; la precaria, que expresa desigualdad e inadecuación; y la social, financiada por el Estado.

Las viviendas rurales construidas con criterios sustentables son asequibles, eficientes energéticamente, reciclan la cosecha, manufacturan de forma responsable los materiales, usan menos agua, promueven la salud de sus habitantes, preservan el hábitat y ecosistemas, promueven la comunidad, son de mayor calidad y su operación es menos costosa (Conelly, 2005).

Hay clasificaciones más específicas, como edificaciones gallegas dedicadas al turismo, que son: castillos, monasterios, casas grandes y rectoras. Las casas de aldea, anteriores a 1940, son rústicas. Las casas de labranza son rústicas pero rurales; en ellas se realizan actividades agropecuarias en las que los huéspedes pueden participar, como actividades de "agroturismo" (García, 2001).

\subsection{Aportaciones al estudio de la vivienda rural por disciplinas}

\section{Medicina y psicología clínica}

Ambas se abocan a la salud, la medicina a la física, y la psicología a la mental. Estas investigaciones aparecen en años recientes y van en aumento por los focos de enfermedad que se han identificado; también detectan problemas de calidad de vida de los habitantes y sus viviendas.

Salud física. Los estudios involucran a grupos vulnerables (niños, mujeres y ancianos). La VR afecta a sus moradores por factores físicos y sociales; los principales problemas son envenenamiento con plomo (derivado de la pintura de casas anteriores a 1978) y asma (Lance, 2000-2001a). La exposición crónica al plomo puede causar daño cerebral, contaminación en la sangre o en el sistema reproductivo, y decrecimiento de funciones motoras y cognitivas (Ryan, 2002). El asma se asocia a factores irritantes como humo, moho, polvo, animales y ácaros (Lance, 2000-2001b); Miller, 2002; Dagoye et al., 2004). Otros problemas en niños rurales son: laceraciones, quemaduras con estufas de madera o calentadores de queroseno, y exposición a elementos calientes como radiadores que se utilizan para calentarse (Lance, 2000-2001a). El agua contaminada y el drenaje en malas condiciones producen diarreas, hepatitis A y deshidratación, además de afectar la higiene personal y la preparación de alimentos (Miller, 2002).

Los problemas de salud en ocasiones son muy localizables por el ecosistema en donde se ubican las viviendas. En las VR de Estados Unidos existe un enemigo común: el moho que se asocia a síntomas asmáticos, daño cerebral y enfermedades respiratorias. Los roedores y sus infecciones impactan la salud de los residentes (Lance, 2000-2001b); Anderson, 2002; Miller, 2002).

El aislamiento geográfico de la VR no facilita la atención de la salud (Muller, Smith, Mellor, Rare \& Genton, 1998); en Nueva Guinea encontraron que a mayor distancia entre las viviendas y los centros de atención a la salud, disminuye la frecuencia de visitas médicas. En Lesotho, África, el espacio exterior de la VR es un recurso agrícola para atender las necesidades de alimentación, sin embargo, 
el estatus nutricional de los niños es bajo (Makhotla \& Hendriks, 2004). En contraposición, en Canadá se encontró como factor protector de contaminantes la distancia entre la VR y las zonas de tráfico vehicular (Miller, Dugandzic, Frescura \& Salares, 2007).

El hacinamiento en la VR se ha convertido en una forma de vida (Miller, 2002). Al compartirse la cocina y el baño entre varias familias, la consecuencia es la propagación de enfermedades como conjuntivitis, resfriado, piojos, humo de segunda mano (fumadores a no fumadores), abuso a menores (unido al uso de drogas y alcohol), violencia doméstica (asociada al estrés por el mismo hacinamiento) y complicaciones con estados depresivos (Miller, 2002; Rural Assistance Center, 2007b).

Salud mental. El estado de la VR es indicador de calidad de vida y sus elementos físicos tienen un impacto importante sobre la salud mental de los residentes (Pereira, Contreras, Guatarasma \& Mejía, 2001; Pérez, 2001). Una pobre calidad de vivienda observada por los indicadores: estructura, privacidad, clima interior, riesgos, higiene y recursos, puede afectar el desarrollo mental de los niños (Evans, Lercher \& Kofler, 2002). En Austria se encontró más afectación en la salud de los niños que vivían en multifamiliares rurales que los de casas independientes (Evans, Saltzman \& Cooperman, 2001). En el área rural de Nueva York se identificó que el acceso visual a elementos naturales desde el interior de la casa proporcionaba a los niños un efecto amortiguador ante los eventos estresantes de sus vidas (Wells \& Evans, 2003); también se encontró que a mayor densidad de habitantes, mayores efectos sobre la salud mental de los niños (Evans, Saegert \& Harris, 2001).

Algunos autores afirman que poseer una vivienda beneficia no sólo la salud mental de los habitantes rurales, sino en general la vida de los residentes. En asentamientos rurales los beneficios incluyen la reducción económica y psicológica de los efectos negativos de las crisis económicas (Lance, 20002001a); también disminuye el hacinamiento y la deserción escolar (Zatz, 20002001); y estabiliza la vida de sus habitantes, ya que pueden conservar el trabajo y se distribuyen mejor los ingresos en la familia (Strauss \& Toney, 2000-2001).

Los eventos violentos dentro de la VR afectan la salud mental de los moradores; éstos se relacionan con abuso de sustancias (Koenig et al., 2003) y factores socioeconómicos (Bates, Schuler, Islam \& Islam, 2004; Angelucci, 2007). Estos autores encontraron que, en general, menores ingresos y menor escolarización, así como el consumo de alcohol, están asociados a la violencia doméstica. Otras investigaciones tratan acerca de lo difícil que es para las mujeres conseguir ayuda en los asentamientos rurales, cuando son víctimas de violencia (Krishnan, Hilbert \& VanLeeuwen, 2001; Rural Assistance Center, 2007a).

La casa también puede proporcionar un contexto social para el abuso de sustancias. Valentine, Holloway, Knell y Jayne (2008), encontraron, en una comunidad rural en Inglaterra, que el consumo de alcohol de los adolescentes en la casa no sólo es tolerado, sino que también era estimulado.

Psicología ambiental. ${ }^{3}$ El hacinamiento en la VR tiene diferentes causas: los poblados ubicados en las sierras tienen casas de pocas habitaciones, en una descansa y duerme la familia, y es frecuente la falta de drenaje y agua potable. En las cercanías a las ciudades, los habitantes rurales adoptan el modelo urbano de vivienda de dos pisos por la disminución de terreno, sin eliminar las actividades agropecuarias; el espacio entre habitaciones y animales, depósitos 
para su comida, insecticidas y fertilizantes, se acorta y se producen focos de contaminación porque se dificulta la limpieza (Sánchez, 2006).

El valor y el significado que tiene la VR para sus habitantes se relaciona con aspectos afectivos, sociales, económicos y utilitarios. En Texcoco, México, el concepto de "casa moderna" asociado a la urbana revela dos formas de valoración y de vida: de progreso, cambio o modernización y conciencia de que su vivienda lo refleja, un estilo, por lo general, de familias que transitan de lo rural a lo urbano. Otras se resisten al cambio y se apegan a la tierra y al territorio (Sánchez, 2009). El significado de residencia cambia paulatinamente, de acuerdo a sentimientos y comportamientos de inclusión o exclusión de los grupos de residentes. Durán y Sánchez (2009) identificaron cinco grados de aprecio por la vivienda y la región, que revelan el proceso de cambio de lo rural a lo urbano, desde la perspectiva psicosocial y cultural.

Derr (2002), en Nuevo México, Núñez y Beasley (2001) en Uruguay, y Pretty, Chipuer y Bramston (2003), en Australia, estudiaron los factores relacionados con el sentido de pertenencia al lugar de residencia ("sentirse en casa"); encontraron como factores asociados: el contacto con la familia y la comunidad, realizar actividades en conjunto (recolección de hierbas y leña), y la percepción y el contacto con elementos naturales del ambiente.

\section{Estudios descriptivos y sociológicos}

La ausencia de sentido de pertenencia puede generar conflictos en las relaciones sociales. Pauli (2008) describe las complicadas relaciones entre las suegras y nueras cuyos esposos son inmigrantes. La obtención de una casa para la nuera, la edificación de una nueva, o la apropiación de otros espacios independientes a la suegra, promueven una diferente estructura en las relaciones familiares.

En Michoacán, México, el retorno del esposo migrante no es siempre favorable para la vida de la esposa en la casa, porque se incrementan las labores domésticas y disminuye la libertad en las actividades (Alvarado \& Núñez, 2006). En una localidad de Nuevo León, México, García (2006) concluyó que las viviendas rurales de esa zona estimulan cada uno de los sentidos de sus habitantes.

Las variables demográficas interaccionan de forma compleja, de tal forma que las relaciones entre los diferentes grupos con la vivienda son muy diversas: con datos estadísticos de Estados Unidos, Hicks (2002) describe las características sociodemográficas de los moradores de los asentamientos rurales relacionadas con aspectos de salud (cantidad de habitantes, médicos, hospitales, dentistas, frecuencia y severidad de enfermedades) que se compararon entre asentamientos con diferentes grados de urbanización. En el mismo país se identificó las variables demográficas de tres grupos: a) de pobladores rurales ancianos confinados en sus casas por motivos médicos (Kalavar \& Rapano, 2003); b) de indigentes sin hogar, hecho que se ha incrementando en las zonas rurales, aunque es un fenómeno no tan visible como en las ciudades (Cloke, Milbourne \& Widdowfield, 2001; Post, 2002). Estas personas son atendidas algunas veces por organizaciones confesionales y comunitarias, proporcionándoles diversos servicios como comida, agua, cuidado de niños, trabajo, etc. (Rural Assistance Center, 2007b); c) los pobres rurales experimentan las peores condiciones de vivienda, son los que menos oportunidades tienen de ser dueños, ya que aunque las casas son más baratas en esas zonas, también lo son los ingresos. Aproximadamente 1,5 millones $(6,6 \%)$ de las viviendas rurales están en muy malas condiciones y el $4 \%$ tienen condiciones de hacinamiento 
(Housing Assistance Council, 2006). A pesar de que el trabajo agrícola es una dimensión importante en Estados Unidos, sus trabajadores son uno de los grupos más pobres del país. Esto se traduce en inhabilidad para poder atender una casa asequible, decente y segura (Housing Assistance Council, 2005).

Los jóvenes tienen diversos problemas para obtener una vivienda o rentarla (Burrows, Ford, Quilgars \& Pleace, 1998; Jones, 2001; Jones, 2004). Al relacionar raza y sexo, se ha reportado que las mujeres rurales tienen menos acceso a las viviendas que los hombres, sobre todo si pertenecen a algunas minorías como afroamericanas o latinas (Allen, 2002). Además, las condiciones de vida y trabajo en la casa son más desfavorables para las mujeres que para los hombres (Núñez \& Beasley, 2001; Robson, 2004).

\subsection{Mejoramiento de la vivienda rural}

Los arquitectos son los profesionales que más aportan para el mejoramiento de la VR, desde aspectos sencillos como la construcción de letrinas (Becerril, 1981) o el uso de materiales económicos (Díaz, 2000), hasta trabajos con teoría elaborada derivada de las ciencias sociales y la psicología para sustentar sus aportes. Promueven una fuerte tendencia a incluir a la población en los proyectos de construcción y a considerar el mejoramiento de los poblados. Los trabajos de agrónomos abordan también aspectos prácticos y hacen consideraciones sobre los problemas de preservación de las culturas e identidades campesinas, y llaman la atención sobre la necesidad de armonizar, en las construcciones de viviendas rurales, la sustentabilidad, el equilibrio ecológico y la calidad de vida.

Aspectos técnicos-arquitectónicos. La tecnología más adecuada para la VR será la que solucione integralmente: gestión, construcción, flexibilidad para crecer, mantenimiento, evolución de la vivienda y su entorno utilitario construido (Rotorando \& Mellace, 2000); y como acciones importantes para mejorarla los autores proponen: a) fortalecer la producción local de materiales y recuperar técnicas tradicionales; b) diseñar en tiempos y costos flexibles adecuados para cada comunidad; c) dar soluciones que respondan a la complejidad y diferenciación social, cultural, tecnológica y territorial, así como a la especificidad de las poblaciones, regiones, recursos, capacidades y naturaleza de las necesidades de los usuarios; d) desarrollar en forma integral el hábitat.

Perspectiva agronómica. La VR debe proveer suficiente protección, higiene, privacidad y comodidad a sus habitantes, y aislarlos de agentes exteriores (calor, frío, lluvia, viento, etc.). Debe estar adecuadamente localizada y tener facilitad de saneamiento, según las actividades agropecuarias que realiza la familia (Sámano et al., 2001). Los autores también llaman la atención sobre el hecho de que el espacio de la VR da alojamiento a rituales y costumbres relacionados con la edificación y la actividad económica y social; de que en ella se realizan distintas actividades en un mismo espacio; de que en su territorio se encuentran las habitaciones, así como corrales, gallineros, depósitos, acequias y canales; y de que todos estos espacios se relacionan con el medio ambiente (clima, agua, catástrofes naturales).

La agronomía aporta también una perspectiva geográfica y ecológica como método indirecto que considera a los usuarios. Para la comprensión de la habitación rural, y para solucionar su diseño y construcción, Guzmán (1991) recomienda una regionalización del país con base en 3 premisas: una región es un instrumento de análisis; es una porción territorial sin límites exactos; conlleva implicaciones generales de representatividad física (clima, altitud y orografía) significativas para el usuario, quien obtiene del medio físico satisfactores para 
diferentes necesidades. En ese marco, es necesario considerar en la VR espacios de actividades domésticas, productivas y de modos de producción.

Perspectiva de la psicología ambiental. Sánchez (2006) siguiere: a) atender operatividad y funcionalidad de la VR, considerando las principales actividades de la familia campesina y las apreciaciones de sus miembros, así como la idea que tienen de las "casas modernas"; y b) que las modificaciones a la VR separen las actividades domésticas de las productivas, invirtiendo en los espacios productivos para acondicionar en ellos lo que los espacios domésticos les proporcionan para sus actividades agropecuarias o de maquila. De esa manera, los espacios internos de las viviendas aun cuando no se modificaran podrían generar mayor bienestar, comodidad y calidad de vida a sus moradores.

Participación de los usuarios. En América Latina la participación de los usuarios se ha requerido por las necesidades de reconstruir pueblos devastados por desastres ambientales, y por el desconocimiento de la cultura regional y la pobreza de los pueblos rurales. Hay diferentes grados de participación; en algunos casos se les interroga, mientras que en otros se investiga de manera indirecta las necesidades familiares y los elementos necesarios para la construcción.

Cuando el planificador no sabe lo que necesita la población en su vivienda, la arquitectura debe buscar, concebir y construir espacios habitables que promuevan el equilibrio ecológico, económico, psicológico y político de los individuos y su sociedad, a partir de una propuesta participativa (Cruz, 2000). Este diseño, de acuerdo a Vargas (2000), permite a la población expresar sus intereses desde las decisiones del proyecto hasta su ejecución, obteniéndose beneficios sociales, políticos, económicos y ambientales. La participación provoca que las personas se identifiquen con el trabajo, lo hagan suyo y se comprometan personalmente; además, que se organicen para resolver sus necesidades y obtener orientación para encontrar soluciones desde los medios que poseen. Es vital que el arquitecto obtenga una visión personal sobre los sentimientos que la población tiene del espacio y sobre la necesidad que se quiere resolver en determinado espacio.

Para la reconstrucción de las viviendas después del huracán Mitch en Nicaragua, pidieron a los usuarios que dibujaran cómo querían la vivienda y se discutieron los aspectos que no deseaban hasta elegir los tres diseños que más les gustaban y convenían (Lagos, 2000). En Mendoza, Argentina, para mejorar las condiciones de vida y favorecer el arraigo de la población rural, se emplearon talleres en los que se decidieron distintos aspectos del diseño; se incentivó al desarrollo de los asentamientos rurales y el uso de fuentes renovables de energía, aportando al desarrollo sustentable (Mitchell, 2000). En Hidalgo, México, para preservar a la VR de cambios que hacen los migrantes al reproducir lo que aprenden en el destino migratorio, se atendió a cada persona que pretendía modificar su vivienda y se explicó su calidad arquitectónica (González, 2000).

Bajo la premisa de que para contribuir al desarrollo rural integral no se debe trabajar en forma asistencialista, sino fortalecer los factores involucrados, Rodulfo et al. (2000) atendieron a productores minifundistas pobres en una zona con propensión a la emigración. Los proyectos habitacionales aumentaron la producción agropecuaria porque se asociaron a la obtención de la vivienda. Si no hay sustentabilidad productiva, la vivienda puede resultar una inversión perdida y la migración es la consecuencia. El proyecto habitacional otorgó financiamiento para materiales de construcción y como contraparte, se fomentó la organización social y la participación de los beneficiarios mediante la mano de obra. Esto 
contribuyó a elevar su autoestima por construir su propia mejora en la calidad de vida; los grupos se fortalecieron con capacitación en oficios y para la producción regional de materiales y técnicas constructivas.

\subsection{Criterios de sostenibilidad y calidad de vida}

Sostenibilidad. Cualquier esfuerzo en materia de vivienda debe basarse en conceptos de sostenibilidad, propiciando el mejoramiento de la calidad de vida de los habitantes y el equilibrio en las dimensiones ecológica, económica y social (Fournier, 2000). Con estos principios, el autor trabajó en Costa Rica con el Programa ID Misión, para participar en la solución del problema habitacional haciendo cambios en la práctica constructiva. Entre los resultados obtenidos hubo: ahorros significativos en el costo y un sistema de construcción integral prefabricado para viviendas y edificaciones livianas, funcionales y estéticas.

En Tesayuca, México, se elaboró un proyecto con ideas sobre la integración de las comunidades al diseño de su hábitat, buscando que fueran sustentables y que a largo plazo garantizaran su utilidad, facilitaran la producción, y disminuyeran la dependencia de los productores del mercado de energía y de acaparadores. Se pretendía conseguir, mediante un diseño arquitectónico adecuado y educación general a los habitantes, que ellos realizaran mejoras a su calidad de vida y a su vivienda (Narváez, 2000). El proyecto se basó en conceptos de planeación territorial, arquitectura y diseño de los espacios abiertos para unidades de producción de leche, diseño de tecnologías baratas de energía alternativa, reducción de desechos y mejoría en la producción de leche; sin embargo, no se tomó en cuenta la cercanía de los productores a su ganado. Este tipo de errores son los que se pueden evitar con la participación de la población beneficiada.

Para la VR en zonas en donde existe traslape entre campo y ciudad, existen recomendaciones para la planificación y el financiamiento económico. En opinión de Vargas (2000), se requiere buscar la sustentabilidad, que evite el arrasamiento de las riquezas forestales, agrícolas y ganaderas, y procure el respeto a la población rural y la creación de la vivienda "rururbana".

Calidad de vida. Un factor que agrava el deterioro de la calidad de vida en los asentamientos rurales es la descomposición ecológica, que hace más desventajosas las condiciones productivas de las familias campesinas (Tena, 2000). Por ello, antes de la calidad de vida en la VR, es necesario considerar las condiciones de los asentamientos rurales. Rodulfo et al. (2000), de Argentina, señalan como principales estrategias para el desarrollo del poblado: 1) innovación en las estrategias de gestión, corresponsabilidad en la formación y aplicación de recursos, y diversificación de las soluciones con ajuste a las necesidades sentidas, en un marco de progresividad y continuidad; 2) las soluciones deben responder a la complejidad y diferenciación social, cultural, tecnológica y territorial, y a la especificidad de las poblaciones, regiones, recursos y capacidades, así como a la naturaleza de sus necesidades.

La necesidad de ofrecer mejores condiciones de vida al trabajador agropecuario exige una nueva estructura en los núcleos poblacionales, con emplazamiento adecuado y aprovechamiento óptimo de los recursos naturales (Pascual, Gómez, Pérez \& López, 2000). También es necesario validar con los usuarios la satisfacción o calidad de vida que les aporta, dado que ésta es, "una sensación existencial, es la percepción que un individuo o comunidad tiene de la calidad de los satisfactores del ambiente en el que vive" (Contreras y Corder, 1994 citados por Carballosa, Cardet \& González, 2002). La calidad ambiental que se percibe 
depende de las características del lugar, teniendo además relación con las experiencias y la cultura de la comunidad.

Criterio de calidad de vida en área rural. Pérez (2001) trabajó cuatro tipos de indicadores de calidad de vida para el medio rural: importancia económica y social que tiene el poblador rural; situación, recursos y cobertura de los servicios de salud; dinámica de la población; y, condiciones ambientales. Justifica su trabajo basándose en la proporción de la población rural en América Latina que representa el $24 \%$ del total y en México representa el 24,5\% (24,398 millones de $98,881)$.

Pereira, Contreras, Guatarasma y Mejía (2001), obtuvieron con metodologías participativas (investigación-acción) en Venezuela, cuatro dimensiones de calidad de vida para una comunidad rural: humana, económica, ambiental y tecnológica.

\section{Modelo de Calidad de Vida de la Vivienda Rural}

Este Modelo permite, por un lado, la visión de conjunto de las relaciones entre disciplinas y sus temas de interés; y por otro, identificar grandes indicadores para la precisión de los elementos que se requieren para la calidad de vida de los moradores de las viviendas rurales. Estos indicadores tienen la característica de multidisciplinariedad, ya que no tienen las limitaciones de las disciplinas con la que cada una ve los requerimientos de la VR. El Modelo presenta en lugar de esa visión de división o de especialidad los niveles sistémicos, con los criterios de Ecología del Desarrollo Humano de Bonfenbrenner (2002).

La característica básica del Modelo es partir de la persona, su familia y su vivienda, avanzando a lo largo de las interacciones del grupo familiar con su vivienda, como espacio construido, y continuando con el medio natural, social y ambiental en donde reside e interactúa, como medio para su desarrollo.

El Cuadro 1 muestra las relaciones verticales y horizontales del Modelo. Las primeras hablan de la inclusión de los niveles más pequeños en los mayores, lo que se observa si se revisan de arriba hacia abajo. El primer lugar es el de las ciencias orientadas directamente a las personas, las familias y sus viviendas; el segundo es para las ciencias sociales; y el tercero para las ambientales. Lo mismo sucede con los niveles ecológicos, al estar comprendidos en el exosistema los dos sistemas anteriores. Las relaciones horizontales de izquierda a derecha indican las concordancias entre ciencias, temas de interés y niveles de sistemas ecológicos de desarrollo humano. 


\section{Cuadro 1. Modelo de Calidad de Vida para la VR.}
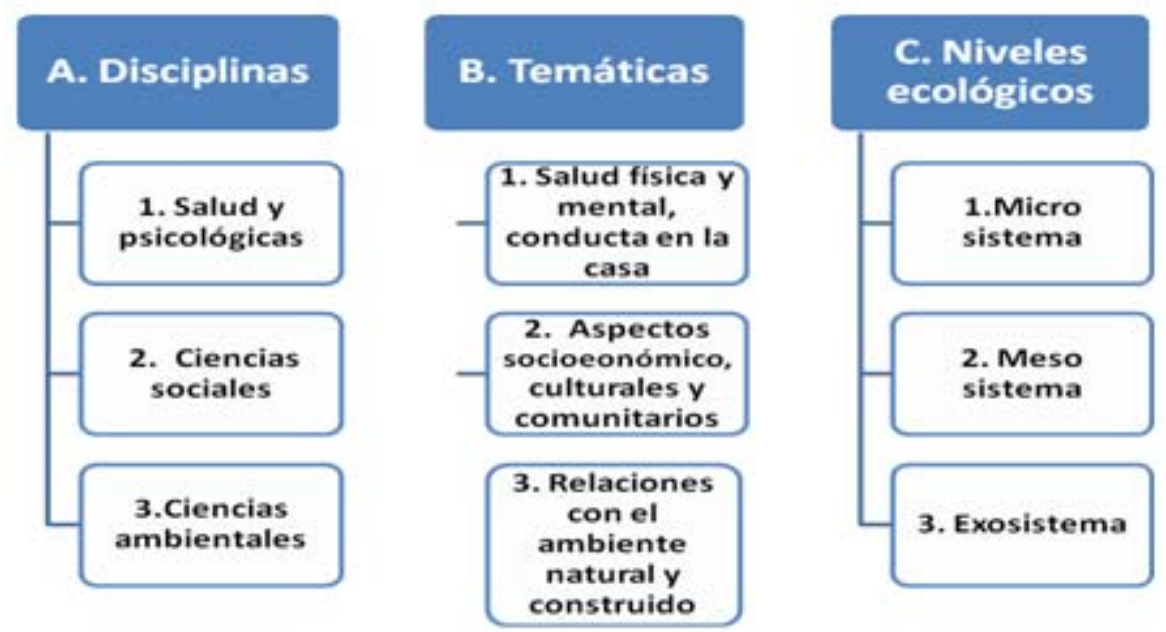

Las ciencias que estudian personas, familias, sus interacciones y el uso de los espacios de sus viviendas se interesan por el sistema más pequeño o microsistema. Las disciplinas cuyos temas de interés son las redes sociales, las costumbres y la cultura comunitaria, así como los problemas que ellas enfrentan en lo económicos y social, se identifican en este Modelo como estudiosas del mesosistema. El nivel más amplio se asigna a las ciencias ambientales, que se interesan por las formas y relaciones que los grupos humanos entablan con el ambiente natural, construido y social, y que abarcan objetivos de dimensiones comunitarias o regionales, constituyendo el exosistema.

Para explicar el Modelo, se divide en dos secciones. El Cuadro 2 contiene dos columnas, A y B: en la A se concentran las disciplinas, y en la B los temas abordados por ellas; el propósito de ambas es mostrar las coincidencias de intereses y sus especificidades. Las dos tienen tres apartados; de la columna A son: A.1) Ciencias de la Salud y Psicológicas, A.2) Ciencias Sociales y A.3) Ciencias Ambientales. Es difícil sustraer a la Psicología Ambiental del contexto social, por lo que se le ubica tanto en A.2 como en A.3. 


\section{Cuadro 2. Disciplinas y Temas de Interés.}
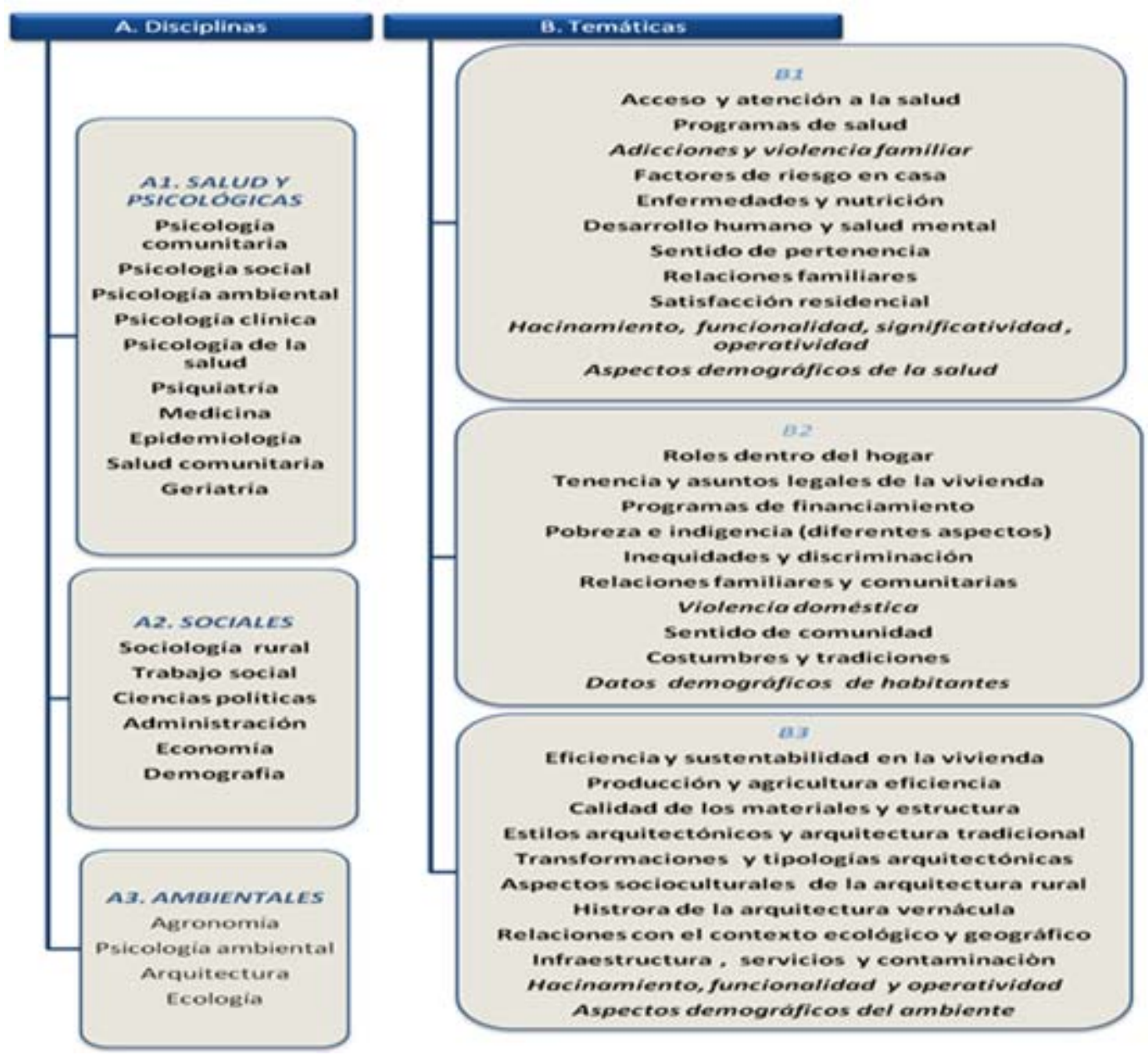

Los tres apartados de la columna B son correspondientes a los de la columna A: B.1) los temas de interés de las Ciencias de la Salud y Psicológicas, B.2) los de las Ciencias Sociales y B.3) de las Ciencias Ambientales. El agrupamiento de ciencias se hizo en función de identificar los conjuntos que más semejanzas tienen en cuanto temáticas estudiadas y concordancias de intereses. Sin embargo, hay interacciones entre conjuntos. Los más sobresalientes son:

- Los datos demográficos son una constante en los tres conjuntos temáticos, porque se convierten en variables relacionadas al tener características de habitantes de la VR, su entorno ecológico y social.

- La violencia intrafamiliar es un tema de interés tanto para las ciencias de la salud y psicológicas, como las sociales.

- El acceso a la salud que se indica en el conjunto B.1, se incluye también en el B.2 en los aspectos de inequidades y discriminación.

- Entre B.2 y B.3 las coincidencias se dan en hacinamiento, funcionalidad y operatividad de la VR, interés manifestado principalmente por psicólogos ambientales y arquitectos. Por su parte, los agrónomos también se interesan por los tres temas, pero con otros nombres, por su injerencia en la productividad agropecuaria y forestal. 
El Cuadro 3 contiene la tercera parte del Modelo, la cual considera una correspondencia entre ciencias, temas y niveles ecológicos de desarrollo humano. De ese ordenamiento se obtiene un primer nivel de indicadores para construir instrumentos que exploren grados de la calidad de vida en las viviendas rurales de manera integral, en territorios delimitados ecológica y geográficamente:

\section{Cuadro 3. Indicadores de calidad de vida por niveles ecològicos.}

\section{Microsistema}

Privacidad, Hacinamiento, Áreas de descanso, Funcionalidad, Operatividad, Relaciones familiares, Ambientes separados entre humanos, animales e implementos agropecuarios, Materiales inocuos, Higiene, Ventilación, Seguridad, Calefacción segura, Materiales y estructura de la casa en óptimas condiciones, Servicios y equipamiento

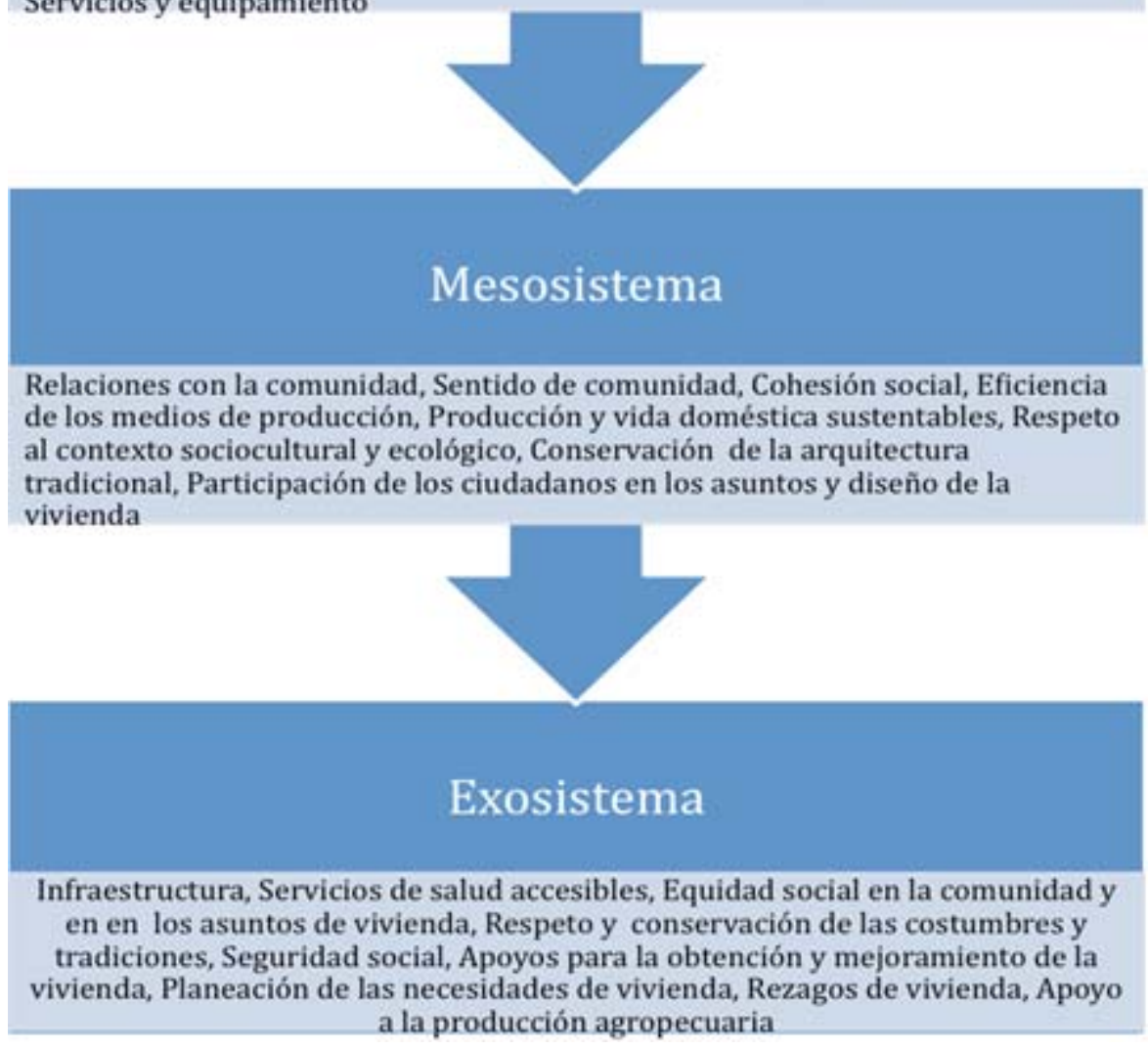

\section{REFERENCIAS}

- Aguilar, B. (2001). Estado del arte sobre la arquitectura vernácula en México. En J. González (Ed.), Vivienda rural y calidad de vida en los asentamientos rurales. Pp. 809-822. Santiago de Cuba: Cyted-Habyted.

- Allen, B. L. (2002). Race and gender inequality in homeownership: Does place make a difference? Rural Sociology, 67(4), 603-621.

- Alvarado, C. y Núñez, M. C. (2006). Malestares emocionales en esposas de migrantes en una comunidad rural del Estado de México. Tesis de Licenciatura inédita, Universidad Nacional Autónoma de México. 
- Anderson, J. (2002). Creating affordable allergen-free housing. Rural Voices, 7(4), 20-22.

- Angelucci, M. (2007). Love on the rocks: Alcohol abuse and domestic violence in rural Mexico. What causes alcohol abuse and domestic violence and how can we stop them? Obtenido el 15 de abril de 2008 desde http://www.iza.org/publications/dps/

- Bates, L. M., Schuler, S. R., Islam, F. e Islam, M. K. (2004). Socioeconomic factors and processes associated with domestic violence in rural Bangladesh. International Family Planning Perspectives, 30(4), 190199.

- Becerril, L. (1981). Datos prácticos de instalaciones hidráulicas y sanitarias. México: IPN.

- Bolis, G. (1982). Las casas campesinas en el Porfiriato. V Memoria y olvido: imágenes de México. México: Martín Casillas.

- Bonfenbrenner, U. (2002). La ecología del desarrollo humano. México: Paidós.

- Burrows, R., Ford, J., Quilgars, D. y Pleace, N. (1998). A place in the country? The housing circumstances of young people in rural England. Journal of Youth Studies, 1(2), 177-194.

- Carballosa, S., Cardet, J. y González, J. (2002). Salud y calidad de vida en el diseño de la vivienda Cubana. Documento presentado en la Quinta reunión de la Red Interamericana de Centros de Salud y Vivienda, Cuba.

- Cardoso, M., A. (1999). Interdisciplina o multidisciplina en el área de la salud. Nueva Época, 7, 31-37.

- Cassils, A. J. (2004, Ene). Overpopulation, Sustainable Development, and Security: Developing and Integrated Estrategy. Population and Environment. U.E. 25(3), 171-194.

- Chayanov, A. V. (1974). La organización de la unidad económica campesina. Buenos Aires: Nueva Visión.

- Cloke, P., Milbourne, P. y Widdowfield, R. (2001). Interconnecting housing, homelessness and rurality: evidence from local authority homelessness officers in England and Wales. Journal of Rural Studies, 17(1), 99-111.

- Conelly, E. F. (2005). Getting started on green affordable housing. Rural Voices, 10(3), 6-7.

- Correa, T. (2000). Conceptualización de la vivienda rural en Panamá. En J. González y M. Villar, (Eds.), II Seminario y taller iberoamericano sobre Vivienda rural y calidad de vida en los asentamientos rurales. (Vol. I, pp. 37-40). México: Universidad Autónoma de San Luis Potosí.

- Cruz, J. F. (2000). La vinculación social de la arquitectura: El diseño participativo. En J. González y M. Villar, (Eds.), II Seminario y taller iberoamericano sobre Vivienda rural y calidad de vida en los asentamientos rurales. (Vol. I, pp. 77-80). México: Universidad Autónoma de San Luis Potosí.

- Dagoye, D., Bekele, Z., Woldemichael, K., Nida, H., Yimam, M., Venn, A. J. et al. (2004). Domestic risk factors for wheeze in urban and rural Ethiopian children. QJMed, 97(8), 489-498.

- Damián, A. (1991). Vivienda rural y autogestión. En V. Guzmán, (Ed.), El medio rural y la producción. (pp. 99-106). México: UAM-X.

- Derr, V. (2002). Children's sense of place in northern New Mexico. Journal of Environmental Psychology, 22(1-2), 125-137.

- Díaz, J. (2000). La vivienda rural en el próximo siglo. En J. González y M. Villar, (Eds.), II Seminario y taller iberoamericano sobre Vivienda rural y calidad de vida en los asentamientos rurales. (Vol. I, pp. 41-48). México: Universidad Autónoma de San Luis Potosí. 
- Durán B. J. y Sánchez Q. C. (2009). El significado de residencia en Xocotlán, municipio de Texcoco, México. En C. Sánchez, (Ed.), Psicología en Ambiente Rural. p 97-117 México, D.F.: Plaza y Valdés.

- Evans, G. W., Lercher, P. y Kofler, W. W. (2002). Crowding and children's mental health: the role of house type. Journal of Environmental Psychology, 22(3), 221-231.

- Evans, G. W., Saegert, S. y Harris, R. (2001). Residential density and psychological health among children in low-income families. Environment and Behavior, 33(2), 165-180.

- Evans, G. W., Saltzman, H. y Cooperman, J. L. (2001). Housing quality and children's socioemotional health. Environment and Behavior, 33(3), 389-399.

- Fournier, Z. R. (2000). Nuevas tecnologías hacia el hábitat sostenible en los asentamientos populares. En J. González y M. Villar, (Eds.), II Seminario y taller iberoamericano sobre Vivienda rural y calidad de vida en los asentamientos rurales. (Vol. I, pp. 274-292). México: Universidad Autónoma de San Luis Potosí.

- García, A. (2001). El territorio de la Palloza. Apuntes para una nueva clasificación tipológica. En J. González, (Ed.), Vivienda rural y calidad de vida en los asentamientos rurales. Pp 59-72. Santiago de Cuba: CytedHabyted.

- (2004). Función del psicólogo en el área rural. Tesis de Licenciatura inédita, Universidad Nacional Autónoma de México.

- García, N. A. (2006). Reseña de La casa campesina y el lugar de los sagrado de Alejandro García García. Ra Ximhai, 2(1), 277-282.

- González, C. (2001). Vivienda rural en México. Vivienda rural y calidad de vida en los asentamientos rurales. Santiago de Cuba: Cyted-Habyted.

- González, J. (2000). El papel de la mujer ante la vivienda rural y la calidad de vida de los asentamientos rurales. En J. González y M. Villar, (Eds.), II Seminario y taller iberoamericano sobre Vivienda rural y calidad de vida en los asentamientos rurales. (Vol. II, pp. 10-11). México: Universidad Autónoma de San Luis Potosí.

- González, R. (2000). Vivienda rural en el municipio de Villa Hidalgo. En J. González y M. Villar, (Eds.), II Seminario y taller iberoamericano sobre Vivienda rural y calidad de vida en los asentamientos rurales. (Vol. II, pp. 168-172). México: Universidad Autónoma de San Luis Potosí.

- Grama, J. L. (2000). Women forgotten: Difficulties faced by rural victims of domestic violence. American Journal of Family Law, 14(3), 173-188.

- Guzmán, V. (1991). El medio rural y la habitación. En V. Guzmán, (Ed.), El medio rural y la producción. (pp. 14-21). México: UAM-X.

- Harte, J. (2007). Human population as a dynamic factor in environmental degradation. Population and Environment, 8, 223-236.

- Heidegger, M. (1992). Hölderlin y la esencia de la poesía. Traducción de Samuel Ramos, publicada en M Heidegger: Arte y Poesía, Buenos Aires, F.C.E.

- Hicks, L. L. (2002). Rural health care. Obtenido el 21 de mayo de 2008, desde http://www.apa.org/rural/hicks.pdf

- Housing Assistance Council. (2005). Farmworker housing: Turning challenges into successes. Rural Voices, 10(2), 1-25.

- (2006). Poverty in rural America. Obtenido el 14 de mayo de 2008, www.ruralhome.org/manager/uploads/Povertyruralamerica.pdf

- Jones, G. (2001). Fitting Homes? Young People's Housing and Household Strategies in Rural Scotland. Journal of Youth Studies, 4(1), 41-62.

- Jones, G. W. (2004). A risky business: experiences of leaving home among young rural women. Journal of Youth Studies, 7(2), 209-220. 
- Kalavar, J. y Rapano, J. (2002). Homebound seniors in rural communities: Recruitment challenges y emerging themes. Journal of Rural Community Psychology, E5 (1). Disponible en: http://www.marshall.edu/jrcp/sp2002/Kalavar.htm, Acceso el: 20 mayo 2008.

- _ (2003). Homebound rural elderly in Pennsylvania: Health perspectives. Journal of Rural Community Psychology, E6(1). Disponible en http://wwww.marshall.edu/jrcp/E6one_Kalavar.htm, Acceso el: 20 mayo 2008.

- Kenneth, C., Land, Lamb y Taylor, A. (2007). Measuring trends in child well-being: An evidence-based approach. Social Indicators Research, 80, 105-132.

- Koenig, M. A., Lutalo, T., Zhao, F., Nalugoda, F., Wabwire-Mangen, F., Kiwanuka, N. et al. (2003). Domestic violence in rural Uganda: evidence from a community-based study. Bulletin of the World Health Organization, 81(1), 53-60.

- Krishnan, S. P., Hilbert, J. C. y VanLeeuwen, D. (2001). Domestic Violence and Help-Seeking Behaviors among Rural Women: Results from a ShelterBased Study. Family y Community Health, 24(1), 28-38.

- Lagos, M. (2000). Las ONGs como puentes de transferencia tecnológica en Iberoamérica. En J. González y M. Villar, (Eds.), II Seminario y taller iberoamericano sobre Vivienda rural y calidad de vida en los asentamientos rurales. (Vol. II, pp. 119-128). México: Universidad Autónoma de San Luis Potosí.

- Llambí, L. (1995). Globalización, Ajuste y Nueva Ruralidad: una agenda para la investigación y el desarrollo rural. Venezuela: Laboratorio de Estudios Rurales y Agrarios.

- $\quad$ Lance, G. (2000-2001a). Why housing matters. Rural Voices, 6(1), 2-3.

- $\quad$ (2000-2001b). Why housing matters to children's well-being. Rural Voices, 6(1), 4-5.

- Landázuri, A. M., Terán, A., Mercado, S. y Sánchez, C. (2003). Habitabilidad interna de la vivienda y calidad de vida. En J. Guevara, (Coord.), Los cambios físicos y sociales de la vivienda popular en Latinoamérica. Cap 1 p. 3-33, Puebla: UPAEP.

- Makhotla, L. y Hendriks, S. (2004). Do home gardens improve the nutrition of rural pre-schoolers in Lesotho? Development Southern Africa, 21(3), 575-581.

- Mellace, F. R. (2000). Tecnología en la vivienda rural: Tucumán, Argentina. En J. González y M. Villar, (Eds.), II Seminario y taller iberoamericano sobre Vivienda rural y calidad de vida en los asentamientos rurales. (Vol. I, pp. 308-316). México: Universidad Autónoma de San Luis Potosí.

- Mercado, S., Ortega, P., Luna, M. y Estrada, C. (1995). Habitabilidad de la Vivienda Urbana. México, D.F.: Facultad de Psicología, UNAM.

- Miller, J. D., Dugandzic, R., Frescura, A.-M. y Salares, V. (2007). Indoor and outdoor derived contaminants in urban and rural homes in Ottawa, Ontario, Canada. Journal of the Air y Waste Management Association, 57(3), 297-302.

- Miller, R. (2002). Danger lurks at home in Indian county. Rural Voices, 7(4), 7-9.

- Mitchell, J. (2000). Diseño de un asentamiento humano en la zona rural del centro oeste de la República Argentina. En J. González y M. Villar, (Eds.), II Seminario y taller iberoamericano sobre Vivienda rural y calidad de vida en los asentamientos rurales. (Vol. II, pp. 179-180). México: Universidad Autónoma de San Luis Potosí. 
- Monterrubio, C. (2000). La mujer y la vivienda rural. Antecedentes de la productividad de la mujer en el medio rural. En J. González y M. Villar, (Eds.), II Seminario y taller iberoamericano sobre Vivienda rural y calidad de vida en los asentamientos rurales. (Vol. II, pp. 43-46). México: Universidad Autónoma de San Luis Potosí.

- Muhajarine, N., Labonte, R., Williams, A. y Randall, J. (2008). Person, perception, and place: What Matters to Health and Quality of Life. Social Indicators Research, 85 (1), 53-80.

- Muller, I., Smith, T., Mellor, S., Rare, L. y Genton, B. (1998). The effect of distance from home on attendance at a small rural health centre in Papua New Guinea. International Journal of Epidemiology, 27(5), 878-884.

- Narváez, A. (2000). Un proyecto para la producción y la vivienda en el ámbito rural. En J. González y M. Villar, (Eds.), II Seminario y taller iberoamericano sobre Vivienda rural $y$ calidad de vida en los asentamientos rurales. (Vol. II, pp. 128-148). México: Universidad Autónoma de San Luis Potosí.

- Núñez, V. y Beasley, A. (2001). Expectativas y sustentabilidad de los pequeños productores rurales en la zona norte de Uruguay. En J. González (Ed.), Memorias del 3er Seminario sobre Vivienda rural y calidad de vida en los asentamientos rurales, (pp. 49-58). Santiago de Cuba: Cyted-Habyted.

- Parada, L. (1993). El concepto de familia. Patrones de distribución del ingreso. En P. Bedolla, O. Bustos y G. Delgado, (Eds.), Estudios de género y feminismo II. (pp. 265-277). México: Fontamara.

- Paredes, M. (2000). La mujer, la vivienda rural y la calidad de vida en los asentamientos rurales. En J. González y M. Villar, (Eds.), II Seminario y taller iberoamericano sobre Vivienda rural y calidad de vida en los asentamientos rurales. (Vol. II, pp. 52-61). México: Universidad Autónoma de San Luis Potosí.

- Parsons, J. (1975). Population and social structure. Bulletin of the British Psychological Society, 28, 248-248.

- Pascual, J. M., Gómez, G., Pérez, I. y López, Y. (2000). Metodología para el estudio de la calidad de vida. Caso de estudio: Asentamiento en Laguna Blanca. En J. González y M. Villar, (Eds.), II Seminario y taller iberoamericano sobre Vivienda rural y calidad de vida en los asentamientos rurales. (Vol. I, pp. 179 - 185). México: Universidad Autónoma de San Luis Potosí.

- Pauli, J. (2008). A house of one's own: Gender, migration and residence in rural Mexico. American Ethnologist, 35(1), 171-187.

- Pereira, N., Contreras, W., Guatarasma, L. y Mejía, N. (2001). En J. González (Ed.), Memorias del 3er Seminario sobre Vivienda rural y calidad de vida en los asentamientos rurales, (pp. 263-274). Santiago de Cuba: Cyted-Habyted.

- Pérez, A. (2001). El medio ambiente, la vivienda rural y la calidad de vida en los asentamientos rurales de Iberoamérica. En J. González (Ed.), Memorias del 3er Seminario sobre Vivienda rural y calidad de vida en los asentamientos rurales, (pp. 237-262). Santiago de Cuba: Cyted-Habyted.

- Pérez, E. (2001). Hacia una Nueva Visión de lo Rural. En N. Giarracca, (Compiladora), ¿Una Nueva Ruralidad en América Latina? 384 pp. Buenos Aires: Colección Grupo de Trabajo Desarrollo Rural.

- Pretty, G. H., Chipuer, H. M. y Bramston, P. (2003). Sense of place amongst adolescents and adults in two rural Australian towns: The discriminating features of place attachment, sense of community and place dependence in relation to place identity. Journal of Environmental Psychology, 23(3), 273-287.

- $\quad$ Post, P. (2002). Hard to reach. Rural Voices, 7(4), 14-16. 
- Redman, Ch. L. y Jones S. N. (2005). The environmental, social, and health dimensions of urban expansion. Population and Environment, 26(6), 505-520

- Robson, E. (2004). Children at work in rural northern Nigeria: patterns of age, space and gender. Journal of Rural Studies, 20(2), 193-210.

- Rodulfo, M. A. B., Calcagno, G., Foce, S., Suárez, M. T., Sabsay, A., Pessano, R. et al. (2000). Vivienda rural y turismo en el Perú. Protección y recuperación del patrimonio vernáculo en moche. En J. González y M. Villar, (Eds.), II Seminario y taller iberoamericano sobre Vivienda rural y calidad de vida en los asentamientos rurales. (Vol. I, pp. 133- 142). México: Universidad Autónoma de San Luis Potosí.

- Rotondaro, R. y Mellace, R. F. (2000). Tecnología en la vivienda rural en Iberoamérica. En J. González y M. Villar, (Eds.), II Seminario y taller iberoamericano sobre Vivienda rural y calidad de vida en los asentamientos rurales. (Vol. I, pp. 243-248). México: Universidad Autónoma de San Luis Potosí.

- Roze, J. (2000). Conceptualización de la vivienda rural. En J. González y M. Villar, (Eds.), II Seminario y taller iberoamericano sobre Vivienda rural y calidad de vida en los asentamientos rurales. (Vol. I, pp. 12-15). México: Universidad Autónoma de San Luis Potosí.

- Rural Assistance Center. (2007a). Domestic violence frequently asked questions. Obtenido el 12 de mayo de 2008, desde http://www.raconline.org/info_guides/public_health/

- $\quad$ (2007b). Frequently asked questions. Obtenido el 12 de mayo de 2008, desde http://www.raconline.org/info_guides/public_health/

- $\quad$ Ryan, D. (2002). Coming to grips, Rural Voices, 7(4), 17-19.

- Rybczynski, W. (1986). La casa, historia de una idea. Madrid: NEREA.

- Sámano, M., Cervera, E., Jiménez, C., Galván, F., Martínez, M., Hernández, F. et al. (2001). Consideraciones sobre la sociedad rural y su desarrollo. En B. Mata y C. Villanueva, (Eds.), México rural: Políticas para su reconstrucción. 393 pp. México: Universidad Autónoma de Chapingo.

- Sánchez, Q. C. (2006, Agosto). Cambios operativos y funcionales en la vivienda rural en zona de expansión demográfica. Psicología para América $\begin{array}{llllll}\text { Latina, } & \text { Número } 7 \text { Agosto } & \text { p } & \text { 1-18 ISSN: } & \text { 1870-350 }\end{array}$ http://psicolatina.org/siete/cambios.html

- $\quad$ (2009). Significatividad de la vivienda rural y los valores de la familia que la habita. Municipio de Texcoco, México. En C. Sánchez, (Ed.). Psicología en Ambiente Rural. p75-96 México, D.F.: Plaza y Valdés.

- Strauss, L. R. y Toney, F. M. (2000-2001). Why housing matters to economic development. Rural Voices, 6(1), 13-14.

- Tena, R. A. (2000). El neoliberalismo en el carnaval. En J. González y M. Villar (Eds.), II Seminario y taller iberoamericano sobre Vivienda rural y calidad de vida en los asentamientos rurales. Pp. 23-36 México: Universidad Autónoma de San Luis Potosí.

- Thomson ISI. (2008). Endnote, Isiresearchsoft. Disponible en www.endnote.com

- Torres, G. (2001). Antecedentes para el atlas de la vivienda rural del Estado de México. En J. González (Ed.), Memorias del 3er Seminario sobre Vivienda rural y calidad de vida en los asentamientos rurales, (pp. 683-690). Santiago de Cuba: Cyted-Habyted.

- Unikel, S. L., Ruiz, Ch. C., y Garza V. (1976). El desarrollo urbano de México: diagnóstico e implicaciones futuras. México: Centro de Estudios Económicos y Demográficos de México.

- Valentine, G., Holloway, S., Knell, C. y Jayne, M. (2008). Drinking places: Young people and cultures of alcohol consumption in rural environments. Journal of Rural Studies, 24(1), 28-40. 
- Vargas, L. (2000). Vivienda rural y calidad de vida en México. En J. González y M. Villar, (Eds.), II Seminario y taller iberoamericano sobre Vivienda rural y calidad de vida en los asentamientos rurales. (Vol. I, pp. 49-66). México: Universidad Autónoma de San Luis Potosí.

- Veenhoven. (2005). Apparent quality of live in nations: How long and happy people lives. Social Indicators Research, 71, 61-86.

- Villalvazo, P., Corona, J. P. y García, S. (2002). Urbano-rural, constante búsqueda de fronteras conceptuales. Notas. Revista de información y análisis, 20, 17-24.

- Villar, M. (2001). Lo valorable en la vivienda rural. En J. González (Ed.), Memorias del 3er Seminario sobre Vivienda rural y calidad de vida en los asentamientos rurales, (pp. 81-90). Santiago de Cuba: Cyted-Habyted.

- Wells, N. M. y Evans, G. W. (2003). Nearby nature: A buffer of life stress among rural children. Environment and Behavior, 35(3), 311-330.

- Zatz, S. (2000-2001). Why housing matters to farmworker health. Rural Voices, 6(1), 5-7.

1. Profesora Investigadora Titular. Colegio de Posgraduados en Ciencias Agrícolas.

2. Posdoctorante. En el Colegio de Posgraduados en Ciencias Agrícolas. Profesor de la Facultad de Arquitectura, de la Universidad Nacional Autònama de Mèxico.

3. Los aportes de la Psicología Ambiental en México se apoyaron en las variables que se estudian de manera sincrónica en varios países y que Mercado, Ortega, Luna y Estrada (1995) han organizado en un Modelo de Habitabilidad de la Vivienda Urbana: operatividad, funcionalidad, significatividad, placer La información que se aporta deriva de una investigación realizada en el municipio de Texcoco, que ha quedado incluido en la Zona Metropolitana de la Ciudad de México. 